\title{
The arcsecond scale properties of 6 UHBLs
}

\author{
Shengxu Yang ${ }^{1}$, Zhongzu $\mathbf{W u}^{1}$, Minfeng $\mathrm{Gu}^{2}$ \\ ${ }^{1}$ College of Science, Guizhou University, Guiyang 550025, China \\ email: zzwu08@gmail.com \\ ${ }^{2}$ Key Laboratory for Research in Galaxies and Cosmology, Shanghai Astronomical \\ Observatory, Chinese Academy of Sciences, \\ 80 Nandan Road, Shanghai 200030, China
}

\begin{abstract}
In this work, by using the VLA archive data, we present the arcsecond scale structure and radio spectrum properties of six UHBLs, which were selected from Nieppola et al. 2006 , including all the UHBLs available with VLA archive data at L,C,X band. Our preliminary result shows that spectrum of UHBLs seems to be steeper than normal HBLs.
\end{abstract}

Keywords. BL Lac objects - galaxies: active - quasars: general

\section{Introduction}

BL Lacs are a class of radio loud active galactic nuclei(AGN), which are characterized by rapid variability, high polarization and non-thermal radiaton. Based on their synchrotron peak frequency, BL Lacs can be divided into four classes: LBLs, IBLs , HBLs, and UHBLs(Padovani \& Giommi 1995; Ghisellini et al. 1999; Giommi et al. 2001). UHBLs are a type of extreme BL Lac objects. It was thought to be extremely faint at radio wavelengths by the dependency of the SED shape and luminosity. Nieppola et al. (2006) found 22 UHBLs, with $\log \nu_{\text {peak }}>19$. Wu et al.(2007) showed that UHBLs have lower radio luminosity and smaller Doppler factor, might be with larger viewing angle. Generally, spectrum index can be an indicator of beaming properties in blazar jets, sources with steeper spectrum index might indicated a less beaming, conversely flater spectrum index shows higher beaming in jets. In this work, we will investigate the spectrum properties of 6 UHBLs.

\section{Observations and Images}

Nieppola et al. (2006) have constructed the SEDs for a large, heterogeneous sample of BL Lacs and this is the first time the SEDs of BL Lacs have been studied with a sample of over 300 objects. In the sample, $22 \mathrm{BL}$ Lacs with $\nu_{\text {peak }}>10^{19} \mathrm{~Hz}$ were classified as UHBLs candidates. From this sample, we searched the archive VLA data for all the UHBLs, there are 6 of them are available with L, C, X band data. In this work, we only present one-epoch data with highest sensitivity for all the 6 UHBLs. The details can be seen in our following paper $\mathrm{Wu}$ et al. 2013. The sample are listed in table 1. Col 1, source name. Col 2, redshift. Col 3, 5, 7, the Epoch of L C X band we have selected, Col 4, 6, 8 the VLA core flux at L C X band separately.

The phase calibration, amplitude calibration and imaging are performed using AIPS software. From the cleaned maps, we measured the core flux density which are listed in Table 1. Based on the one-epoch core flux, we construct radio spectra for six UHBLs by the multi-frequency flux densities measured. The spectral shape is assumed to be a power law. The slopes were computed with the Least Square Method and are reported in Table 1. 
Table 1. The six UHBLs from Nieppola et al. 2006

\begin{tabular}{lccrrrrrr}
\hline source & $\mathrm{z}$ & Epoch $_{L}$ & $f_{c, L}(\mathrm{mJy})$ & Epoch $_{C}$ & $f_{c, C}(\mathrm{mJy})$ & Epoch $_{X}$ & $f_{c, X}(\mathrm{mJy})$ & $\alpha$ \\
\hline 1ES 0229+200 & 0.14 & 2002.36 & 42.18 & - & $45.0^{*}$ & 1998.21 & 35.32 & 0.07 \\
2E 0323+0214 & 0.147 & 1991.65 & 68.25 & 1991.43 & 45.43 & 1990.38 & 24.67 & 0.5 \\
2ES 0414+0057 & 0.287 & 1985.28 & 82.62 & 1995.55 & 64.79 & 1998.37 & 24.88 & 0.615 \\
EXO 0706+591 & 0.125 & 2002.15 & 66.81 & 1993.15 & 52.07 & 1995.55 & 45.54 & 0.21 \\
PG 1218+304 & 0.184 & 1987.65 & 67.22 & 1997.42 & 59.31 & 1995.62 & 53.34 & 0.129 \\
2E 1415+2557 & 0.237 & 1985.59 & 84.04 & 1992.76 & 46.9 & 2009.80 & 32.43 & 0.519 \\
\hline \hline
\end{tabular}

Notes: (1)* form Perlman et al. (1996)
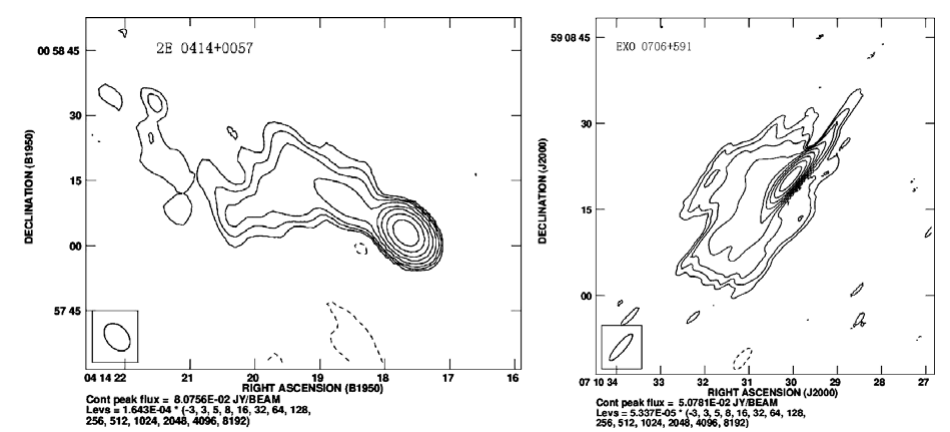

Figure 1. The VLA structure of UHBLs

\section{Results and discussions}

We construct radio spectra for six UHBLs, we find that 3 of 6 UHBLs could have a steep spectrum such as 2E 0323+0214,2ES 0414+0057,2E 1415+2557 and others could have a flat spectrum. In comparison with previously studied results of normal HBLs(Cavallotti et al. 2004), the spectral index of UHBLs seems larger than normal HBLs. From their arescond scale images, we find that most of them show core+halo and core+jet structure.

\section{Acknowledgements}

This work is supported by the NSFC grants (No. 10978009, 11163002, 10833002, 11073039, and 10803015), and by the 973 Program (No. 2009CB824800).

\section{References}

Cavallotti, F., Wolter, A., Stocke, J. T., \& Rector, T. 2004, A\&A, 419, 459

Ghisellini, G. 1999, ApLEGC, 39, 17

Giommi, P., Ghisellini, G., Padovani, P., \& Tagliaferri, G. 2001, AIPC, 599, 441

Nieppola, E., Tornikoski, M., \& Valtaoja, E. 2006, A\&SA, 445, 441

Padovani, P. \& Giommi, P. 1995, ApJ, 446, 547

Perlman, E. S., Stocke, J. T., Schachter, J. F., et al. 1996, ApJS, 104, 251

Wu, Z. Z., Jiang, D. R., Gu, M. F., \& Liu, Y., 2007, A\&SA, 466, 63

$\mathrm{Wu}, \mathrm{Z} . \mathrm{Z} ., \mathrm{Gu}, \mathrm{M}$. F., Jiang, D. R., in preparation 